OPEN ACCESS

Edited by:

Roland Wohlgemuth,

Lodz University of Technology, Poland

Reviewed by:

Elvira Mass,

University of Bonn, Germany

John Lough,

Medical College of Wisconsin,

United States

*Correspondence:

Mingming Fang

dafeifang@163.com

${ }^{\dagger}$ These authors have contributed equally to this work

Specialty section:

This article was submitted to

Molecular Medicine,

a section of the journal

Frontiers in Cell and Developmental

Biology

Received: 06 October 2019 Accepted: 22 May 2020

Published: 18 June 2020

Citation:

Yang Y, Yang $G, Y u L$, Lin L, Liu L, Fang $M$ and $X u Y$ (2020) An Interplay

Between MRTF-A and the Histone

Acetyltransferase TIP60 Mediates

Hypoxia-Reoxygenation Induced

iNOS Transcription in Macrophages.

Front. Cell Dev. Biol. 8:484.

doi: 10.3389/fcell.2020.00484

\section{An Interplay Between MRTF-A and the Histone Acetyltransferase TIP60 Mediates Hypoxia-Reoxygenation Induced iNOS Transcription in Macrophages}

\author{
Yuyu Yang ${ }^{1,3,6 t}$, Guang Yang ${ }^{5 t}$, Liming Yüt, Lin Lin ${ }^{1+}$, Li Liü ${ }^{4}$, Mingming Fang ${ }^{2,3 *}$ and \\ Yong $X u^{3,4}$
}

\begin{abstract}
' Jiangsu Key Laboratory for Molecular and Medical Biotechnology, College of Life Sciences, Nanjing Normal University, Nanjing, China, ${ }^{2}$ Center for Experimental Medicine, Jiangsu Health Vocational College, Nanjing, China, ${ }^{3}$ Institute of Biomedical Research, Liaocheng University, Liaocheng, China, ${ }^{4}$ Key Laboratory of Targeted Intervention of Cardiovascular Disease and Collaborative Innovation Center for Cardiovascular Disease, Department of Pathophysiology, Nanjing Medical University, Nanjing, China, ${ }^{5}$ Department of Pathology, Suzhou Municipal Hospital Affiliated With Nanjing Medical University, Suzhou, China, ${ }^{6}$ Key Laboratory of Emergency and Trauma of Ministry of Education, Institute of Cardiovascular Research of the First Affiliated Hospital, Hainan Medical University, Haikou, China
\end{abstract}

Cardiac ischemia-reperfusion injury (IRI) represents a major pathophysiological event associated with permanent loss of heart function. Several inter-dependent processes contribute to cardiac IRI that include accumulation of reactive oxygen species (ROS), aberrant inflammatory response, and depletion of energy supply. Inducible nitric oxide synthase (iNOS) is a pro-inflammatory mediator and a major catalyst of ROS generation. In the present study we investigated the epigenetic mechanism whereby iNOS transcription is up-regulated in macrophages in the context of cardiac IRI. We report that germline deletion or systemic inhibition of myocardin-related transcription factor A (MRTF-A) in mice attenuated up-regulation of iNOS following cardiac $I R I$ in the heart. In cultured macrophages, depletion or inhibition of MRTF-A suppressed iNOS induction by hypoxia-reoxygenation (HR). In contrast, MRTF-A over-expression potentiated activation of the iNOS promoter by HR. MRTF-A directly binds to the iNOS promoter in response to HR stimulation. MRTF-A binding to the iNOS promoter was synonymous with active histone modifications including trimethylated $\mathrm{H} 3 \mathrm{~K} 4$, acetylated $\mathrm{H} 3 \mathrm{~K} 9, \mathrm{H} 3 \mathrm{~K} 27$, and H4K16. Further analysis revealed that MRTF-A interacted with H4K16 acetyltransferase TIP60 to synergistically activate iNOS transcription. TIP60 depletion or inhibition achieved equivalent effects as MRTF-A depletion/inhibition in terms of iNOS repression. Of interest, TIP60 appeared to form a crosstalk with the H3K4 trimethyltransferase complex to promote iNOS trans-activation. In conclusion, we data suggest that the MRTF-A-TIP60 axis may play a critical role in iNOS transcription in macrophages and as such be considered as a potential target for the intervention of cardiac IRI.

Keywords: transcriptional regulation, epigenetics, macrophages, cardiac ischemia-reperfusion injury, iNOS 


\section{INTRODUCTION}

Cardiac ischemia, following such incidents as major surgeries (e.g., organ transplatation) or thrombosis, poses significant threat to the heart, and the survival of the organism. Attempts to resuscitate the ischemic heart can be met with restoration of the cardiac function but often, paradoxically, worsen the structural and functional loss of the myocardium and dampen the prognosis of the patients (Eltzschig and Eckle, 2011). This critical pathophysiological event, termed ischemia-reperfusion injury (IRI), is thought to be programmed by a series of independent yet inter-connected processes. Reactive oxygen species (ROS), for instance, become excessively produced, and/or inefficiently removed inflicting extensive damages to major macrobiomolecules (Granger and Kvietys, 2015). IRI is also accompanied by increased leukocyte infiltration and aberrant inflammatory response (Lutz et al., 2010). In addition, mitochondrial dysfunction during IRI not only promotes ROS generation but contributes to ATP depletion causing energy shortage (Tompkins et al., 2006). These processes are often paralleled by changes in gene expression patterns in the heart, characterized by up-regulation of enzymes involved in ROS production (e.g., NADPH oxidase), and pro-inflammatory mediators (Amberger et al., 2002).

Inducible NO synthase (iNOS) is a prototypical proinflammatory mediator that can be robustly up-regulated by a range of stimuli including hypoxia-reoxgenation (HR) in macrophages, which is often mediated by NF-кB (Griscavage et al., 1996; Buxade et al., 2012). In addition to its pivotal role in the inflammatory response, iNOS also serves as a major catalyst for ROS production during IRI (Granger and Kvietys, 2015). Previously it has been shown that germline deletion of iNOS in mice protects against IRI in the heart (Marfella et al., 2004), in the kidneys (Ling et al., 1999), and in the gut (Suzuki et al., 2000). Following IRI, iNOS expression is elevated in the heart (Ding et al., 2005). The epigenetic mechanism whereby IRI promotes iNOS transcription is not clear.

Myocardin-related transcription factor A (MRTF-A) is a multifaceted transcriptional modulator. MRTF-A is ubiquitously expressed and is dispensable for embryonic development. Postnatally, MRTF-A has been shown to participate in an array of pathophysiological processes including tissue fibrosis (Small et al., 2010; Fan et al., 2015; Tian et al., 2015; Xu et al., 2015), sepsis (Yu et al., 2017a), colitis (Yu et al., 2014), pulmonary hypertension (Chen et al., 2015), cardiac hypertrophy (Weng et al., 2015a), and cancer metastasis (Cheng et al., 2015). Although MRTF-A was initially identified as a cofactor for serum response factor (SRF), later investigations have indicated that MRTF-A can interact with other sequence-specific transcription factors (TFs) such as NF- $\kappa B$ (Fang et al., 2011), Sp1 (Luchsinger et al., 2011), and Smad3 (Morita et al., 2007) and regulate the transcriptional events mediated by these TFs. It has recently been reported by our laboratory that MRTF-A is essential for the pathogenesis of cardiac IRI in mice relying on a mechanism in which MRTF-A activates the transcription of NADPH oxidases (Yu et al., 2018). Building on this discovery, we investigated the regulation of iNOS transcription in the context of cardiac IRI by MRTF-A. We report that MRTF-A contributes to iNOS transcription in macrophages by interacting with the histone acetyltransferase TIP60. Therefore, the MRTF-A-TIP60 axis may represent an attractive target in the development of novel therapeutic solutions against cardiac IRI.

\section{MATERIALS AND METHODS}

\section{Cell Culture, Plasmids, Transient Transfection, and Reporter Assay}

RAW264 cells were maintained in DMEM supplemented with $10 \%$ FBS. Mouse bone marrow derived macrophages (BMDMs) were isolated and differentiated as previously described (Yu et al., 2017a). Primary cardiac macrophages were purified from the non-myocyte suspension by magnetic beads coated with anti-F4/80 antibody (Miltenyi Biotech). Hypoxia-reoxygenation (HR) was performed as previously described (Ren et al., 2013; $\mathrm{Yu}$ et al., 2018). Briefly, macrophages were exposed to $1 \%$ $\mathrm{O}_{2}$ in a hypoxia chamber (Pro-Ox Model C21, BioSpherix, Parish, NY, United States) for $3 \mathrm{~h}$ followed by reoxygenation in a regular cell-culture incubator with ambient $21 \% \mathrm{O}_{2}$ for 9 h. MRTF-A expression constructs (Li et al., 2018c), iNOS promoter-luciferase construct (Crosby et al., 2005), ASH2 expression construct (Wu et al., 2008), and TIP60 expression construct (Lin et al., 2012) have been previously described. Small interfering RNAs were purchased from Dharmacon. Transient transfection was performed with Lipofectamine 2000. Cells were harvested $48 \mathrm{~h}$ after transfection and reporter activity was measured using a luciferase reporter assay system (Promega) as previously described (Fan et al., 2017; Yu et al., 2017b; Yang Y. et al., 2018). CCG-1423 (S7719) and MG149 (S7476) were purchased from Selleck.

\section{Animals}

All animal experiments were reviewed and approved by the Ethics Committee on Humane Treatment of Laboratory Animals of Nanjing Medical University (Reference\#: 1709013). Germline MRTF-A knockout mice (KO; Sun et al., 2006) and macrophage conditional MRTF-A mice (CKO; Yu et al., 2018) have been described previously. To induce cardiac IRI, the mice were anesthetized with a mixture of ketamine $(120 \mathrm{mg} / \mathrm{kg})$, and xylazine $(6 \mathrm{mg} / \mathrm{kg})$. Following left thoracotomy, the left anterior descending coronary artery was ligated with a 6-0 silk ligature over a $1 \mathrm{~mm}$ polyethylene tube (PE-10) for $45 \mathrm{~min}$ before reperfusion. The control mice were sham operated wherein the ligature around the LAD was not tied. The mice were sacrificed $24 \mathrm{~h}$ after the surgery.

\section{Protein Extraction, Immunoprecipitation, and Western Blot}

Whole cell lysates were obtained by re-suspending cell pellets in RIPA buffer (50 mM Tris pH7.4, $150 \mathrm{mM} \mathrm{NaCl}$, and 1\% Triton X$100)$ with freshly added protease inhibitor (Roche) as previously described (Zeng et al., 2018; Li et al., 2018e; Fan et al., 2019). Nuclear proteins were extracted essentially as described before (Li et al., 2018d). Antibodies were incubated with cell lysates overnight before being absorbed by Protein A/G-plus Agarose 
beads. Precipitated immune complex was released by boiling with 1X SDS electrophoresis sample buffer. Western blot analyses were performed with anti-MRTF-A (Santa Cruz, sc-32909), anti-Tip60 (Santa Cruz, sc-166323), anti-iNOS (Santa Cruz, sc-651), and anti- $\beta$-actin (Sigma, A2228) antibodies. Image J software was used for densitometrical quantification and densities of target proteins were normalized to those of $\beta$-actin. Data are expressed as relative protein levels compared to the control group which is arbitrarily set as 1 .

\section{RNA Isolation and Real-Time PCR}

RNA was extracted with the RNeasy RNA isolation kit (Qiagen, Germantown, MD, United States) as described before (Kong et al., 2019a,b; Li et al., 2019a). Reverse transcriptase reactions were performed using a SuperScript First-strand Synthesis System (Invitrogen, Waltham, MA, United States). Real-time PCR reactions were performed on an ABI Prism 7500 system with the following primers: mouse $\quad M r t f-a$, 5' -CCCAAAGGTAGCAGACAGTTC-3' and $5^{\prime}$-GAGTGGGTGATATGGAGGTGG-3'; mouse iNOS, $5^{\prime}$-CAGAGGACCCAGAGACAAGC- $3^{\prime}$ and $5^{\prime}$-TGCTGAAACA TTTCCTGTGC-3'; and mouse Tip60, 5'-GCCTGGACGGA AGCGGAAATCTAAT- $3^{\prime}$ and $5^{\prime}$-AAACACTTGGCCAGAAGA CACAG- $3^{\prime}$. Ct values of target genes were normalized to the $\mathrm{Ct}$ values of a housekeekping control gene (18s, 5'-CGCGGTTCTATTTTGTTGGT-3' and 5'-TCGTCTTCGA AACTCCGACT- $3^{\prime}$ ) using the $\Delta \Delta$ Ct method and expressed as relative mRNA expression levels compared to the control group which is arbitrarily set as 1 .

\section{Chromatin Immunoprecipitation (ChIP)}

Chromatin immunoprecipitation assays were performed essentially as described before (Liu et al., 2018, 2019a,b; Zeng et al., 2018; Zhang et al., 2018; Li et al., 2018a-e, 2019b-f; Yang Y. et al., 2018; Yang et al., 2019a,b; Fan et al., 2019; Lu et al., 2019; Shao et al., 2019; Weng et al., 2019; Zhao et al., 2019; Kong et al., 2019a,b). Briefly, chromatin was cross-linked with 1\% formaldehyde. DNA was fragmented into 500 bp pieces using a Branson 250 sonicator (30\% output power; 6 cycles of 10 s sonication +10 s intermission). Aliquots of lysates containing $200 \mu \mathrm{g}$ of protein were used for each immunoprecipitation reaction with anti-MRTF-A (Santa Cruz, sc-32909), anti-Tip60 (Santa Cruz, sc-166323), anti-trimethyl H3K4 (Millipore, 07473), anti-acetyl H3K9 (Millipore, 07-352), anti-acetyl H3K27 (Millipore, 07-360), anti-acetyl H4K16 (Millipore, 07-328), anti-ASH2 (Bethyl Laboratories, A300-489A), or pre-immune IgG. Precipitated DNAs were amplified with the following primers: Nos2 promoter, 5'-AGAGTGATGTAATCAAGCAC-3' and $5^{\prime}$-AAAGTTGTGACCCTGGCAG-3'; Gapdh promoter, $5^{\prime}$-ATCACTGCCACCCAGAAGACTGTGGA-3' and $5^{\prime}$ CTCATACCAGGAAATGAGCTTGACAAA - $3^{\prime}$.

\section{In vitro HMT Assay}

The HMT assay was performed as previously described (Wu et al., 2008). Precipitated immune complex was mixed with histone H3 (Millipore, Kankakee, IL, United States), S-adenosyl methionine (SAM, Sigma), BSA, and MAB buffer (50 mM Tris $\mathrm{pH}$ 8.5, $20 \mathrm{mM} \mathrm{KCl}, 10 \mathrm{mM} \mathrm{MgCl} 2,10 \mathrm{mM} \beta$-mercaptoethanol, and $250 \mathrm{mM}$ sucrose). After incubation at $37^{\circ} \mathrm{C}$ overnight, SDS loading buffer was added to stop reactions, and the methylation of histone $\mathrm{H} 3$ was determined by Western blotting.

\section{Statistical Analysis}

For comparison between two groups, two-tailed, unpaired Student's $t$-test was performed. For comparison between more than two groups, one-way ANOVA with post hoc Scheffe analyses were performed using an SPSS package. Unless otherwise specified, $P$ values smaller than 0.05 were considered statistically significant.

\section{RESULTS}

\section{MRTF-A Deficiency Attenuates Ischemia-Reperfusion Induced iNOS Expression in Mice}

We have previously showed that MRTF-A promotes cardiac IRI in mice (Yu et al., 2018). Since iNOS activation has been implicated in the pathogenesis of cardiac IRI, we asked whether MRTF-A might contribute to iNOS transcription in this process. To this end, 8-week male wild type (WT), and MRTF-A KO mice were subjected to cardiac IRI. As shown in Figures 1A,B, iNOS levels were elevated in the heart following IRI; the induction of cardiac iNOS was much more modest in the KO mice than in the WT mice. Next, we injected the mice with an MRTFA inhibitor CCG-1423 before exposing them to the cardiac IRI. Similar to MRTF-A deletion, MRTF-A inhibition attenuated iNOS induction in the heart (Figures 1C,D).

In order to examine the effect of macrophage-specific deletion of MRTF-A, the Mrtfa-flox mice were crossbred with the Lyz2-Cre mice to generate constitutive macrophage conditional MRTF-A knockout (CKO) mice (Yu et al., 2018). We then compared the levels of iNOS in CKO mice and WT mice. Again, qPCR, and Western blotting demonstrated that loss of MRTF-A in macrophages was sufficient to dampen iNOS induction in the heart following IRI (Figures 1E,F). When F4/80+ macrophages were isolated from the heart following the IR procedure, iNOS expression was down-regulated in cell isolated from the $\mathrm{KO}$ mice compared to the WT mice (Figure 1G). Similarly, iNOS expression was reduced in $\mathrm{F} 4 / 80^{+}$macrophages isolated from the mice injected with CCG as opposed to those isolated from the mice injected with saline (Figure 1H). Together, these data suggest that MRTF-A might play a role activating macrophagederived iNOS in response to ischemia-reperfusion.

\section{MRTF-A Deficiency Attenuates Hypoxia-Reoxygenation Induced iNOS Expression in Macrophages}

Based on the observation that MRTF-A deficiency correlated with down-regulation of iNOS expression in the heart, we hypothesized that MRTF-A might contribute to iNOS transcription in response to HR. To test this hypothesis, the following experiments were performed. RAW264 cells were transfected with two separate pairs of siRNAs targeting MRTF-A followed by exposure to HR. HR-induced iNOS expression 
A

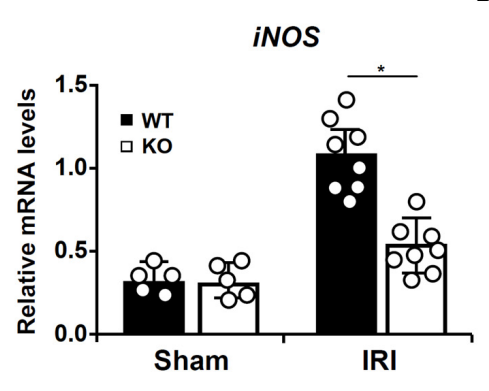

B
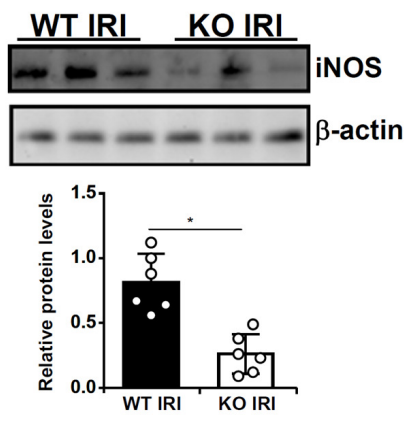

C

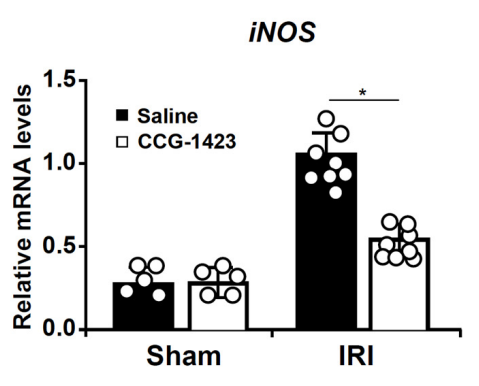

\section{Saline IRI CCG IRI}
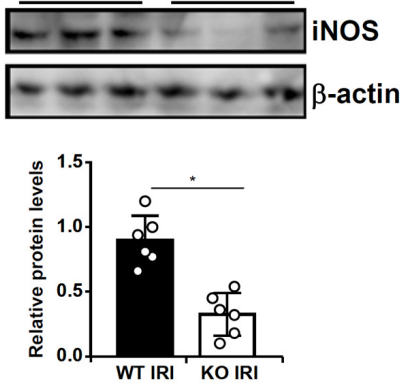

E

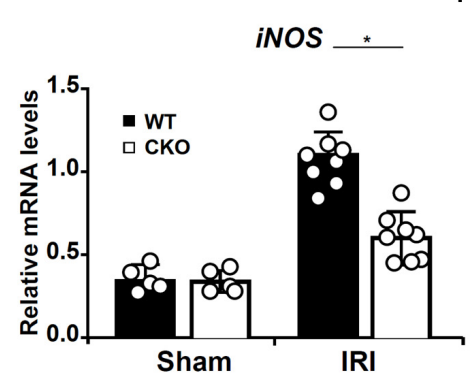

WT IRI CKO IRI
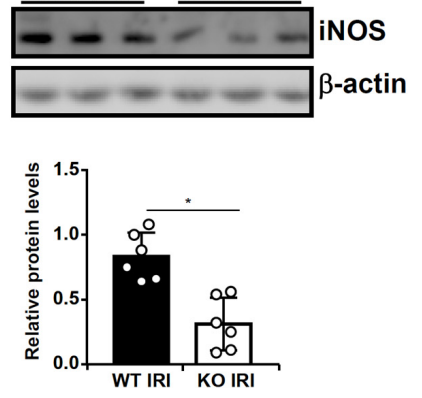

G

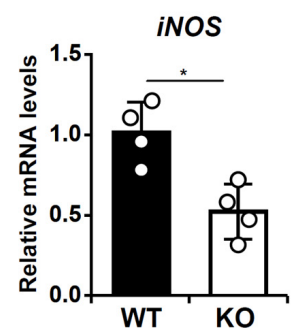

H

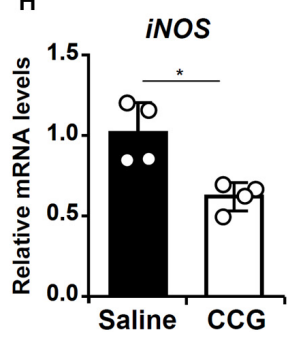

FIGURE 1 | MRTF-A deficiency attenuates ischemia-reperfusion induced iNOS expression in mice. (A,B) Wild type (WT) or MRTF-A knockout (KO) mice were subjected to cardiac ischemia-reperfusion injury or the sham procedure as described in Methods. Expression levels of iNOS in the heart were examined by qPCR and Western. $\mathrm{N}=5-8$ mice for each group. (C,D) C57/BL6 mice were injected with CCG-1423 (1 mg/kg) daily for 2 weeks before the cardiac ischemia-reperfusion procedure as described in Methods. Expression levels of iNOS in the heart were examined by qPCR and Western. N = 5-8 mice for each group. (E,F) Wild type (WT) or macrophage conditional MRTF-A knockout (CKO) mice were subjected to cardiac ischemia-reperfusion injury or the sham procedure as described in Methods. Expression levels of iNOS in the heart were examined by qPCR and Western. $\mathrm{N}=5-8$ mice for each group. (G) Wild type (WT) or MRTF-A knockout (KO) mice were subjected to cardiac ischemia-reperfusion injury as described in Methods. F4/80+ macrophages were isolated and expression levels of iNOS were examined by qPCR. $\mathrm{N}=4$ mice for each group. (H) C57/BL6 mice were injected with CCG-1423 for 2 weeks before the cardiac ischemia-reperfusion procedure as described in Methods. $\mathrm{F} 4 / 80^{+}$macrophages were isolated and expression levels of iNOS were examined by $\mathrm{qPCR}$. $\mathrm{N}=4 \mathrm{mice}$ for each group. 
was significantly down-regulated by MRTF-A knockdown (Figures 2A,B). Similarly, treatment of RAW264 cells with CCG1423 dose-dependently suppressed HR-induced iNOS expression (Figures 2C,D). Finally, primary BMDMs were isolated from WT and KO mice. HR stimulation provoked iNOS expression more robustly in WT cells than in KO cells (Figures 2E,F).

\section{MRTF-A Binds to the iNOS Promoter to Activate iNOS Transcription}

NOS2 (iNOS) transcription can be activated by a host of sequence-specific TFs including NF- $\mathrm{B}$ (Xie et al., 1993) and AP1 (Lowenstein et al., 1993), both of which have been found to interact with MRTF-A (Fang et al., 2011; Weng et al., 2015a). We therefore asked whether MRTF-A could directly regulate iNOS transcription in response to HR. To this end, a reporter construct fused to the proximal iNOS promoter (Crosby et al., 2005) was transfected into HEK293 cells. HR stimulated the iNOS promoter activity and MRTF-A over-expression greatly potentiated induction of the iNOS promoter by HR (Figure 3A). In contrast, a dominant negative (DN) MRTF-A suppressed the induction of the iNOS promoter activity by HR stimulation (Figure 3B). Similarly, HR-induced iNOS promoter activity was diminished by CCG-1423 treatment (Figure 3C). Next, ChIP assay performed in RAW cells (Figure 3D), and BMDMs (Figure 3E) confirmed that occupancy of MRTF-A on the iNOS promoter was greatly enhanced when the cells were exposed to HR stimulation; by comparison, no significant binding of MRTFA was detected on the GAPDH promoter. Of note, a complex between MRTF-A and NF- $\kappa$ B, a sequence-specific transcription factor known to activate iNOS transcription, was detected on the iNOS promoter, but not the GAPDH promoter, following the HR stimulation (Figures 3F,G), suggesting that MRTF-A might be recruited by $\mathrm{NF}-\kappa \mathrm{B}$.

\section{MRTF-A Modulates Histone Modifications Surrounding the iNOS Promoter}

When macrophages were exposed to HR stimulation, the iNOS promoter became abounded with trimethylated H3K4, a marker for active chromatin regions, which was consistent with its transcriptional activation; MRTF-A silencing attenuated H3K4 trimethylation (Figure 4A). It was also observed that MRTFA depletion suppressed H3K9 acetylation (Figure 4B), H3K27 acetylation (Figure 4C), and H4K16 acetylation (Figure 4D) on the iNOS promoter. Similarly, CCG-1423 treatment partially blocked the accumulation of trimethyl H3K4 (Figure 4E), acetyl H3K9 (Figure 4F), acetyl H3K27 (Figure 4G), and acetyl H4K16 (Figure 4H). In addition, when both WT and KO BMDMs were exposed to HR stimulation, accumulation of trimethyl H3K4 (Figure 4I), acetyl H3K9 (Figure 4J), acetyl H3K27 (Figure 4K), and acetyl H4K16 (Figure 4L) on the iNOS promoter was much more modest in KO cells than in WT cells. Of note, there was no significant change in overall histone levels ( $\mathrm{H} 3$ or $\mathrm{H} 4)$ on the iNOS promoter with or without the HR challenge suggesting that histone eviction/deposition may not participate in the regulation of iNOS transcription in the present experimental settings (Supplementary Figure S1).

\section{TIP60 Interacts With MRTF-A to Activate iNOS Transcription}

We decided to focus on the H4K16 acetylation because its role in the regulation of pro-inflammation transcription is comparably under-appreciated. In mammalian cells, the MYST family of proteins, consisting of TIP60, hMOF/MYST1, MOZ, HBO1, and MORF, are considered dedicated H4K16 acetyltransferases (Avvakumov and Cote, 2007); TIP60 and hMOF/MYST1 are the only two members of this family that have been confirmed to possess H4K16 acetyltransferase activity thus far (Taipale et al., 2005; Tang et al., 2013). We have previously published a study in which compelling evidence argues for a role of hMOF in the pathogenesis of cardiac IRI (Yu et al., 2018). Therefore, we focused on TIP60 in the regulation of iNOS transcription. Evidence presented below suggests that MRTF-A might recruit TIP60 to activate iNOS transcription. ChIP assay showed that TIP60 occupied the same region of the iNOS promoter as MRTFA (Figure 5A). Co-immunoprecipitation experiments showed that MRTF-A and TIP60 were in the same complex in HEK293 cells (Figure 5B) and RAW cells (Figure 5C). More importantly, Re-ChIP assay showed that HR stimulation enhanced the interaction between MRTF-A and TIP60 on the iNOS promoter (Figure 5D). Functionally, over-expression of TIP60 activated the iNOS promoter synergistically with MRTF-A (Figure 5E). A small-molecule inhibitor of TIP60 (MG149) suppressed the induction of iNOS expression by HR (Figures 5F,G). In addition, TIP60 knockdown blocked iNOS induction (Figures 5H,I), and erased the accumulation of H4K16 acetylation on the iNOS promoter (Figure 5J). Of intrigue, binding of MRTF-A to the iNOS promoter was attenuated without TIP60 (Figure 5K).

\section{A Crosstalk Between TIP60 and COMPASS Contributes to iNOS Transcription}

An interesting observation was that TIP60 depletion in HRtreated macrophages led to the erasure of $\mathrm{H} 3 \mathrm{~K} 4$ trimethylation on the iNOS promoter (Figure 6A). This observation prompted us to investigate the possibility that TIP60 might form a crosstalk with the $\mathrm{H} 3 \mathrm{~K} 4$ methyltransferase complex. In mammals, H3K4 methylation is catalyzed by the complex of proteins associated with Set1 (COMPASS; Shilatifard, 2012). COMPASS is a multiprotein complex consisting of common structural/regulatory subunits (e.g., ASH2, WDR5, and Rbbp5) and distinct catalytic subunits (MLL1, MML2, MLL3, MLL4, SET1A, and SET1B). In vitro HMT assay was performed to test this hypothesis. As shown in Figure 6B, antibodies targeting ASH2, a key component of COMPASS, and TIP60 both precipitated an H3K4 methyltransferase activity. Furthermore, ASH2-associated H3K4 methyltransferase activity was significantly dampened when TIP60 was depleted by siRNAs (Figure 6C). Co-expression of TIP60 and ASH2 additively augmented the iNOS promoter activity (Figure 6D). These data suggest that a crosstalk between TIP60 and COMPASS may contribute to iNOS transcription. 
A

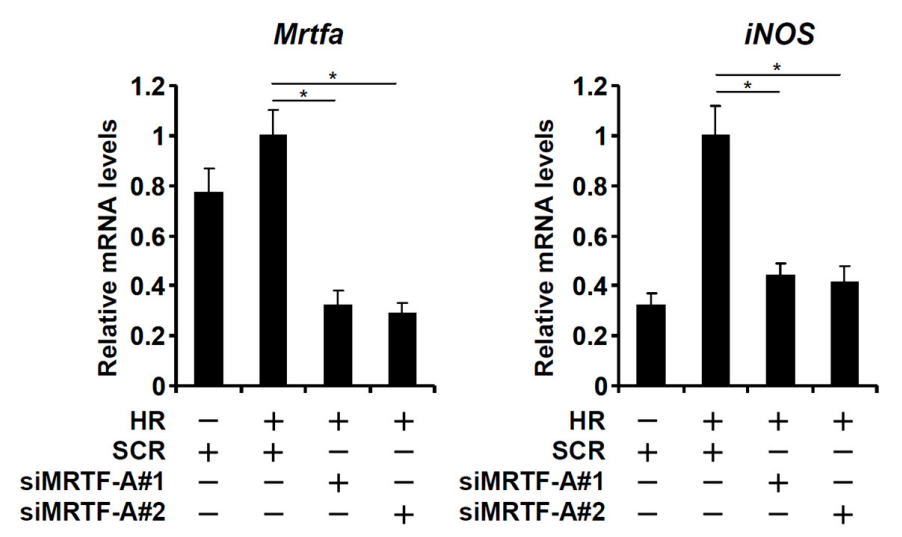

B

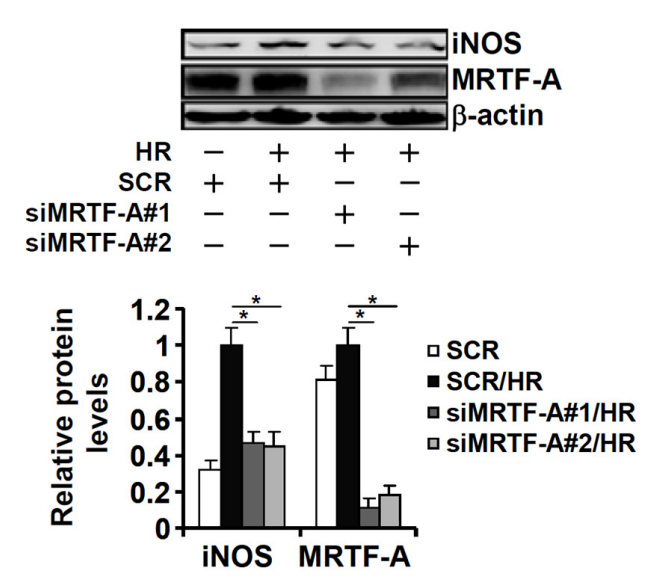

C

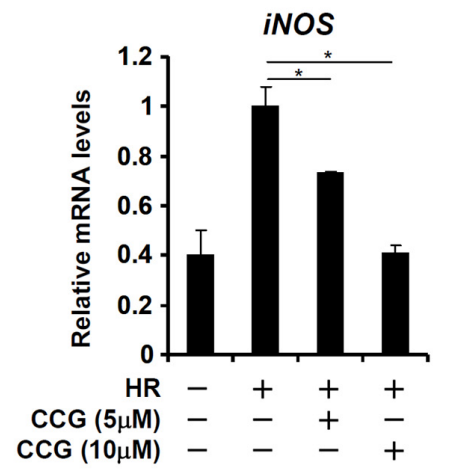

E

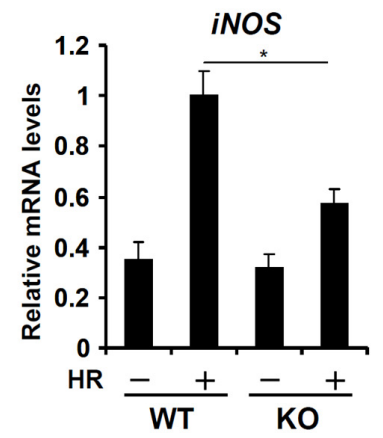

D

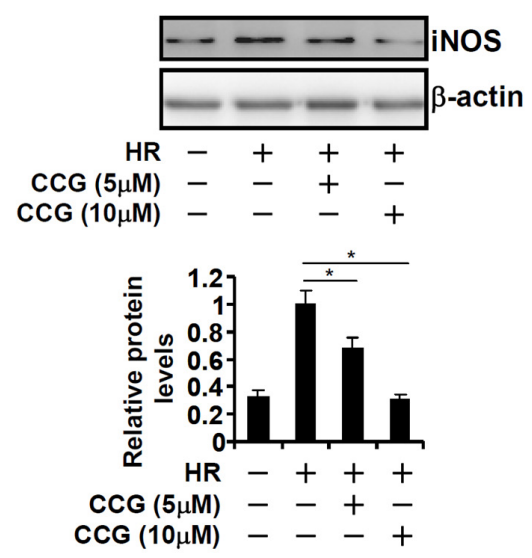

F

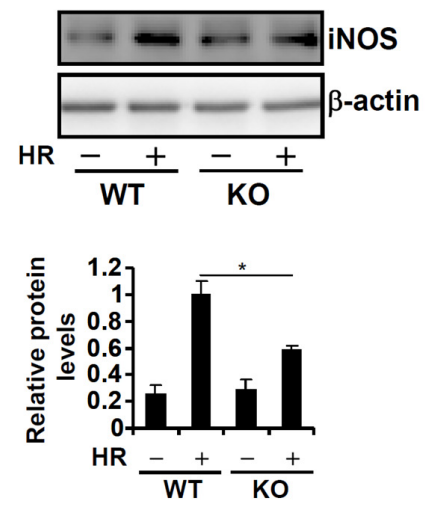

FIGURE 2 | MRTF-A deficiency attenuates hypoxia-reoxygenation induced iNOS expression in macrophages. (A B) RAW264 cells were transfected with siRNA targeting MRTF-A or scrambled siRNA (SCR) followed by exposure to hypoxia-reoxygenation. Expression levels of iNOS were examined by qPCR and Western. (C,D) RAW264 cells were treated with CCG-1423 and/or hypoxia-reoxygenation. Expression levels of iNOS were examined by qPCR and Western. (E,F) Primary BMDMs were isolated from WT and MRTF-A KO mice and exposed to hypoxia-reoxygenation. Expression levels of iNOS were examined by qPCR and Western. $\mathrm{N}=3$ for all the experiments. Data represent averages of three independent experiments and error bars represent SEM. 
A

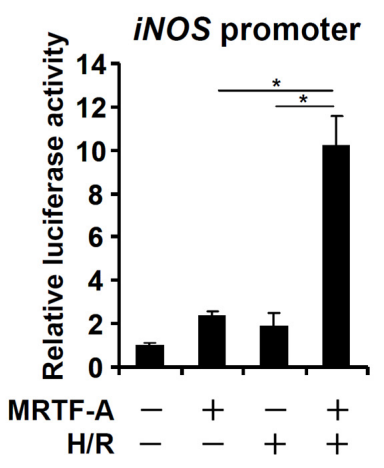

B

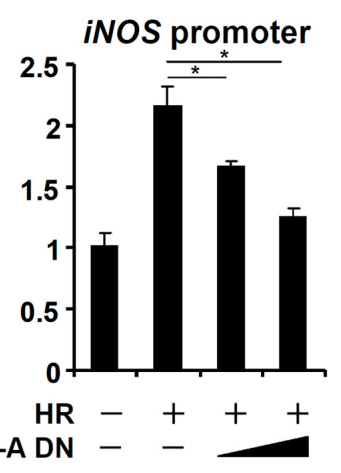

C

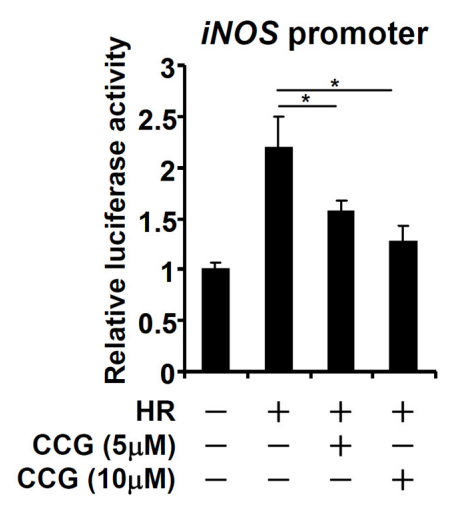

D

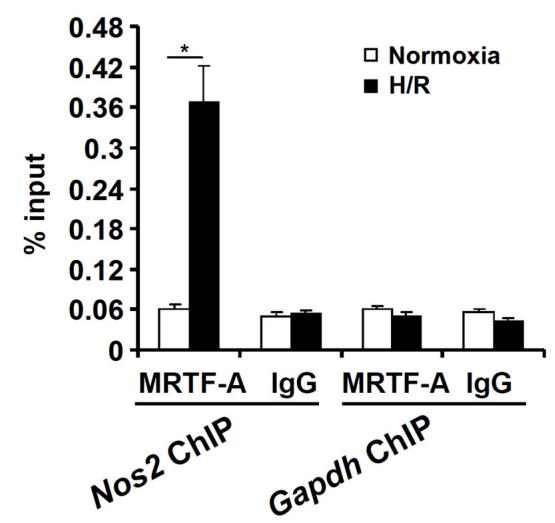

E

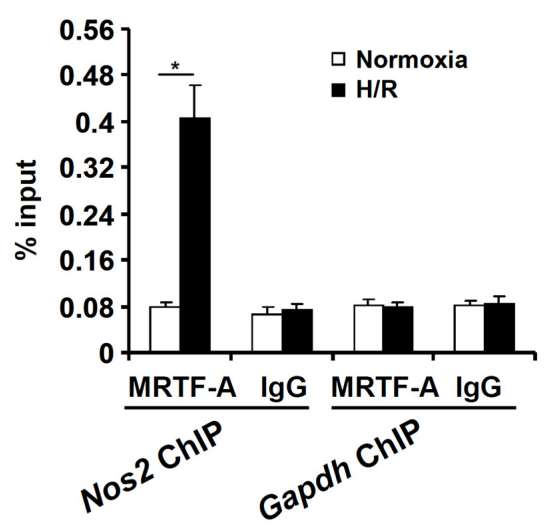

G

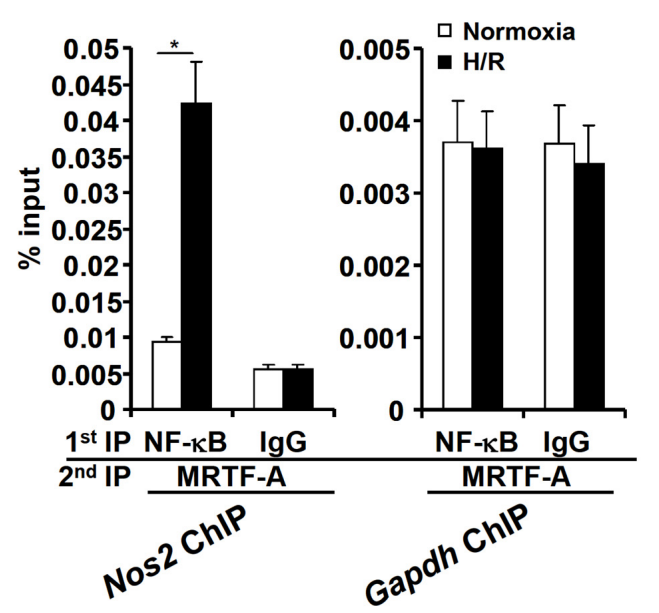

FIGURE 3 | MRTF-A binds to the iNOS promoter to activate iNOS transcription. (A) An iNOS promoter construct was transfected into HEK293 cells with or without MRTF-A followed by exposure hypoxia-reoxygenation. Luciferase activities were normalized by protein concentration and GFP fluorescence. (B) An iNOS promoter construct was transfected into HEK293 cells with or without MRTF-A DN followed by exposure hypoxia-reoxygenation. Luciferase activities were normalized by protein concentration and GFP fluorescence. (C) An iNOS promoter construct was transfected into HEK293 cells followed by treatment with CCG-1423 and/or hypoxia-reoxygenation. Luciferase activities were normalized by protein concentration and GFP fluorescence. (D,E) RAW264 cells (D) or primary BMDMs (E) were exposed to hypoxia-reoxygenation. ChIP assays were performed with anti-MRTF-A or IgG. $(\mathbf{F}, \mathbf{G})$ RAW264 cells $\mathbf{( F )}$ or primary BMDMs $(\mathbf{G})$ were exposed to hypoxia-reoxygenation. Re-ChIP assays were performed with indicated antibodies. $\mathrm{N}=3$ for all the experiments. Data represent averages of three independent experiments and error bars represent SEM. 


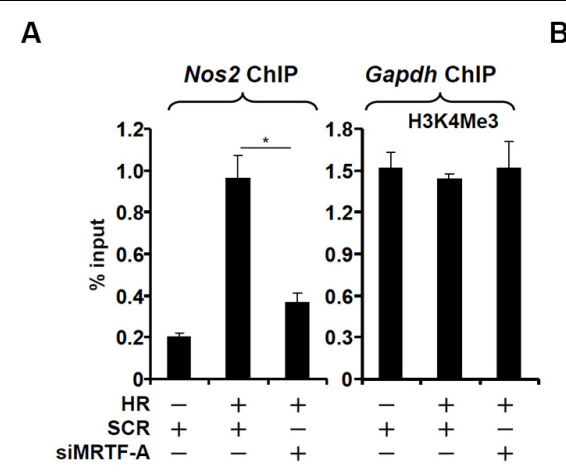

D

B

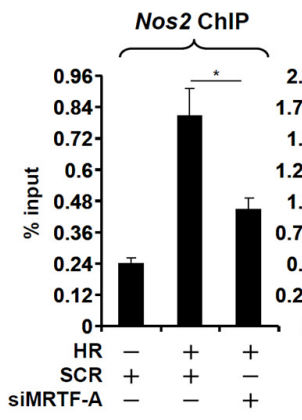

E

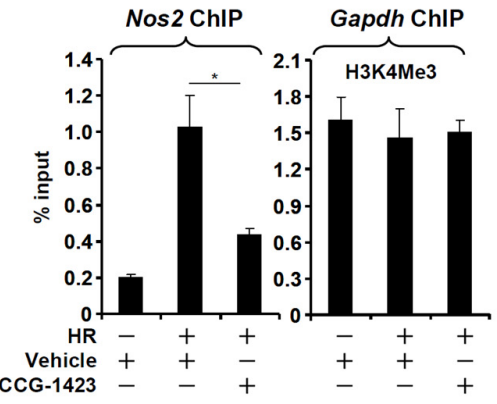

C

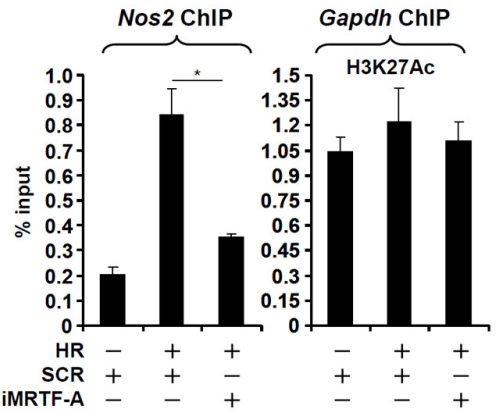

F

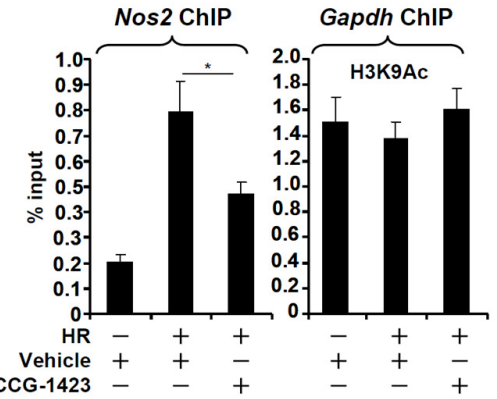

G

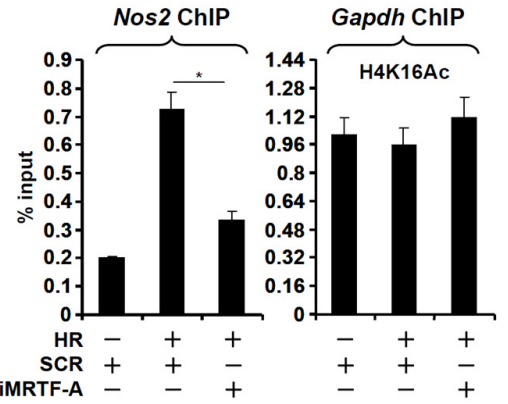

H

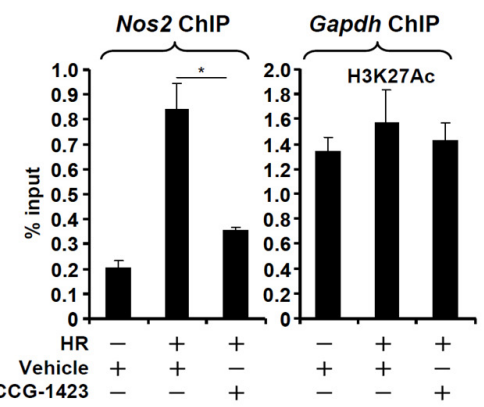

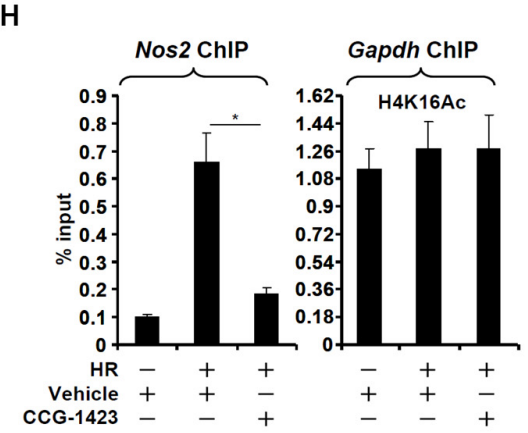

I

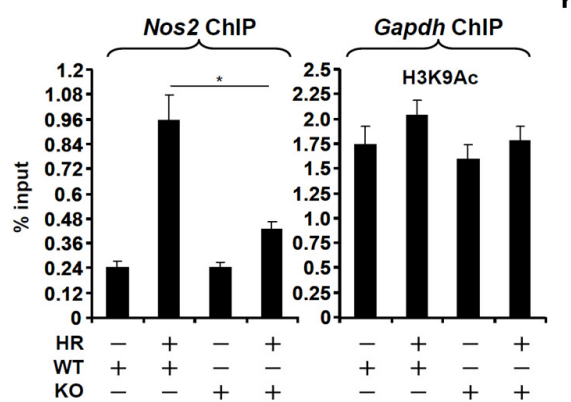

K

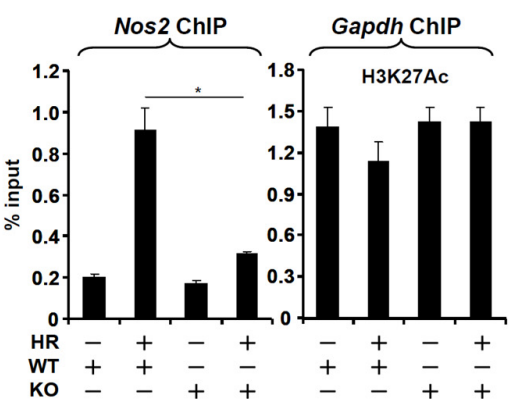

L

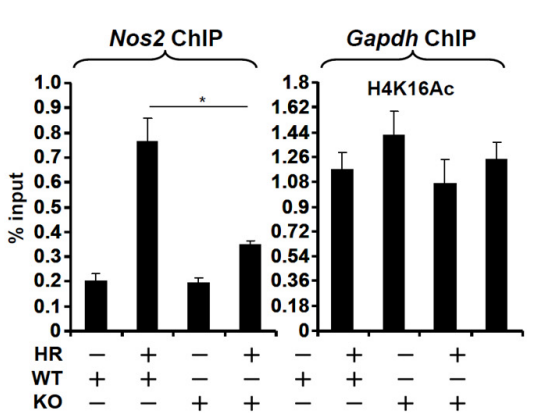

FIGURE 4 | MRTF-A modulates histone modifications surrounding the iNOS promoter. (A-D) RAW264 cells were transfected with siRNA targeting MRTF-A or SCR followed by exposure to hypoxia-reoxygenation. ChIP assays were performed with anti-acetyl H3 (A), anti-acetyl H3K9 (B), anti-acetyl H3K27 (C), and anti-acetyl H4K16 (D). (E-H) RAW264 cells were treated with CCG-1423 and/or hypoxia-reoxygenation. ChIP assays were performed with anti-acetyl H3 (E), anti-acetyl H3K9 (F), anti-acetyl H3K27 (G), and anti-acetyl H4K16 (H). (I-L) Primary BMDMs were isolated from WT and MRTF-A KO mice and exposed to hypoxia-reoxygenation. ChIP assays were performed with anti-acetyl H3 (I), anti-acetyl H3K9 (J), anti-acetyl H3K27 (K), and anti-acetyl H4K16 (L). N = 3 for all the experiments. Data represent averages of three independent experiments and error bars represent SEM. 
A
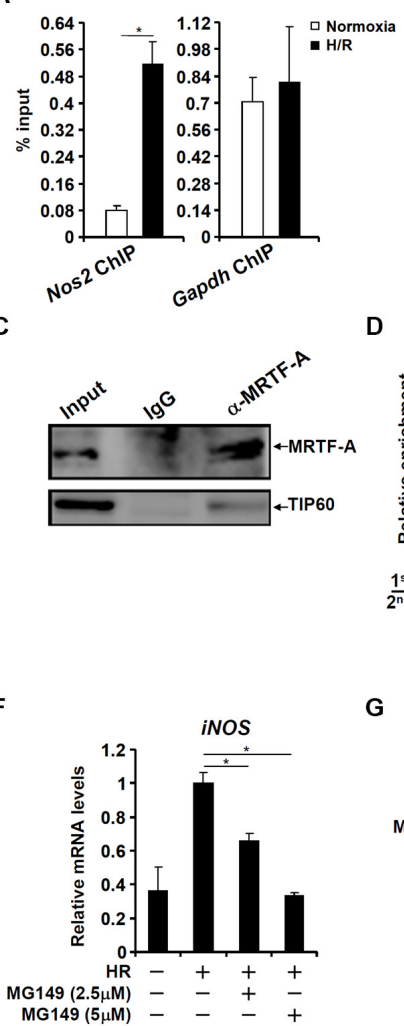

B

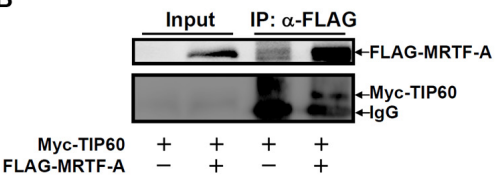

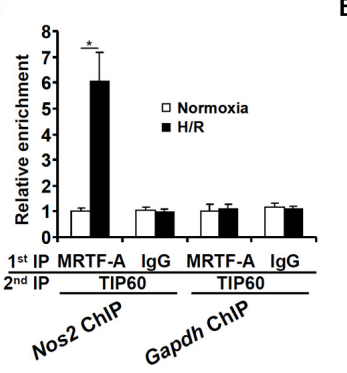

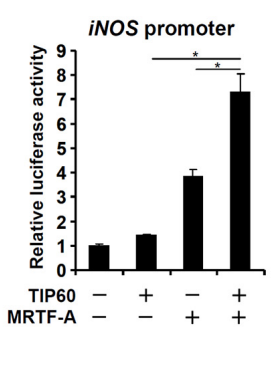

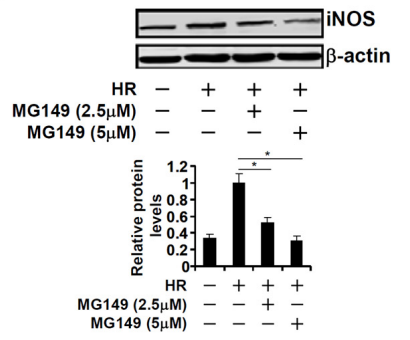

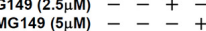

H

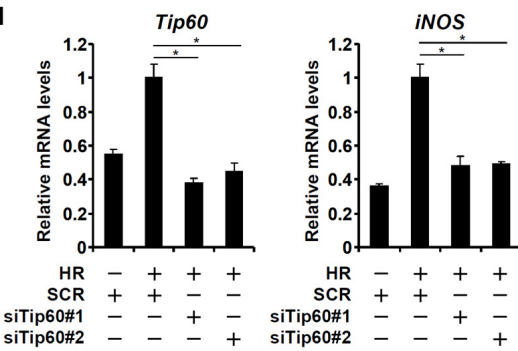

I

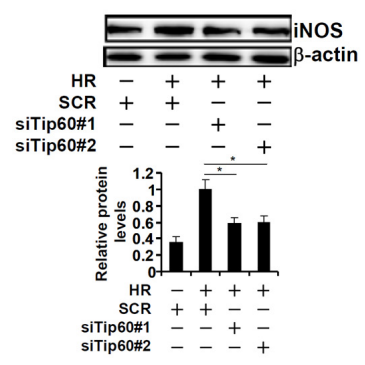

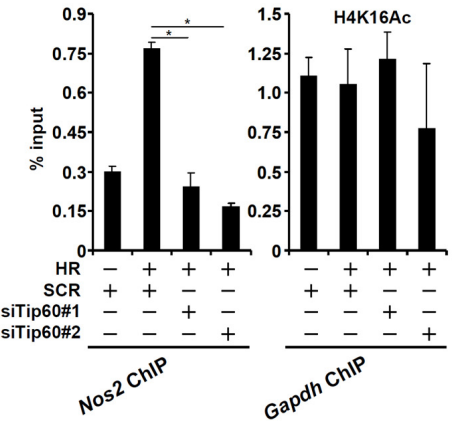

K

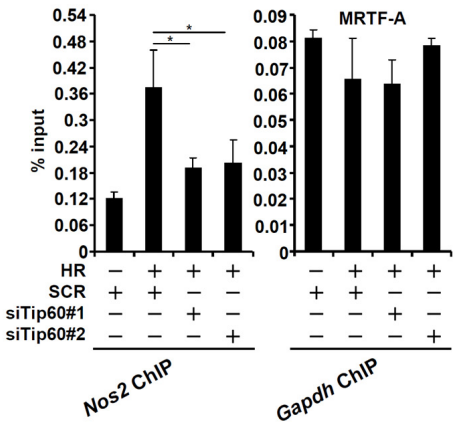

FIGURE 5 | TIP60 interacts with MRTF-A to activate iNOS transcription. (A) RAW264 cells were exposed to hypoxia-reoxygenation. ChIP assays were performed with anti-TIP60 or IgG. (B) HEK293 cells were transfected with FLAG-MRTF-A and/or Myc-tagged TIP60. Immunoprecipitation was performed with anti-FLAG. (C) Whole cell lysates from RAW cells were immunoprecipitated with anti-MRTF-A or IgG. (D) RAW264 cells were exposed to hypoxia-reoxygenation. Re-ChIP assays were performed with indicated antibodies. (E) An iNOS promoter construct was transfected into HEK293 cells with MRTF-A and/or TIP60. Luciferase activities were normalized by protein concentration and GFP fluorescence. (F,G) RAW264 cells were treated with MG149 and/or hypoxia-reoxygenation. Expression levels of iNOS were examined by qPCR and Western. (H-K) RAW264 cells were transfected with siRNA targeting TIP60 or scrambled siRNA (SCR) followed by exposure to hypoxia-reoxygenation. Expression levels of iNOS were examined by qPCR and Western. ChIP assays were performed with anti-acetyl H4K16 and anti-MRTF-A. N = 3 for all the experiments. Data represent averages of three independent experiments and error bars represent SEM. 
A

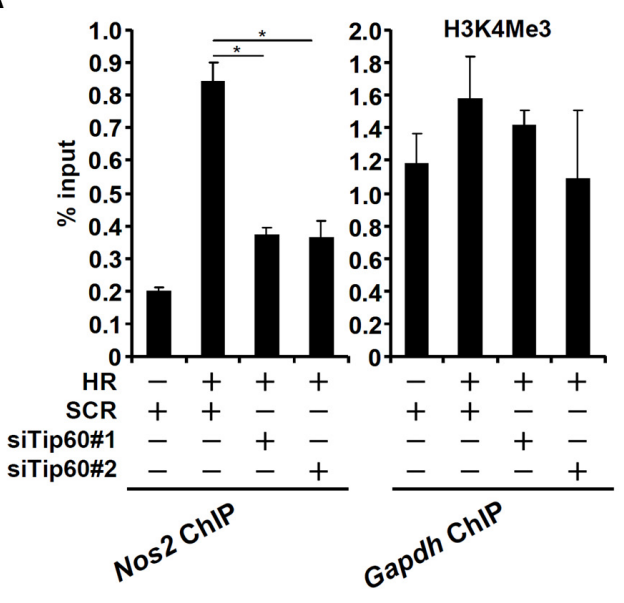

C
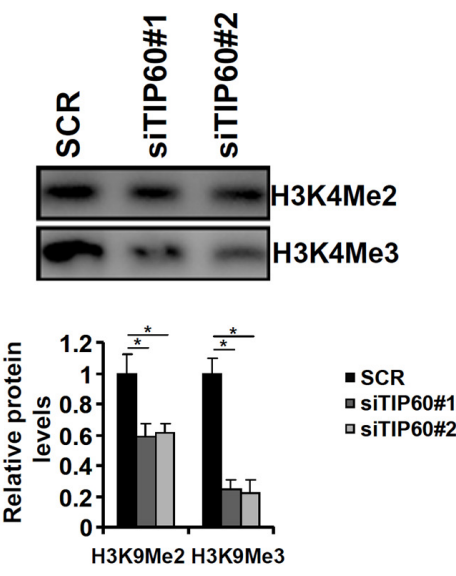

B
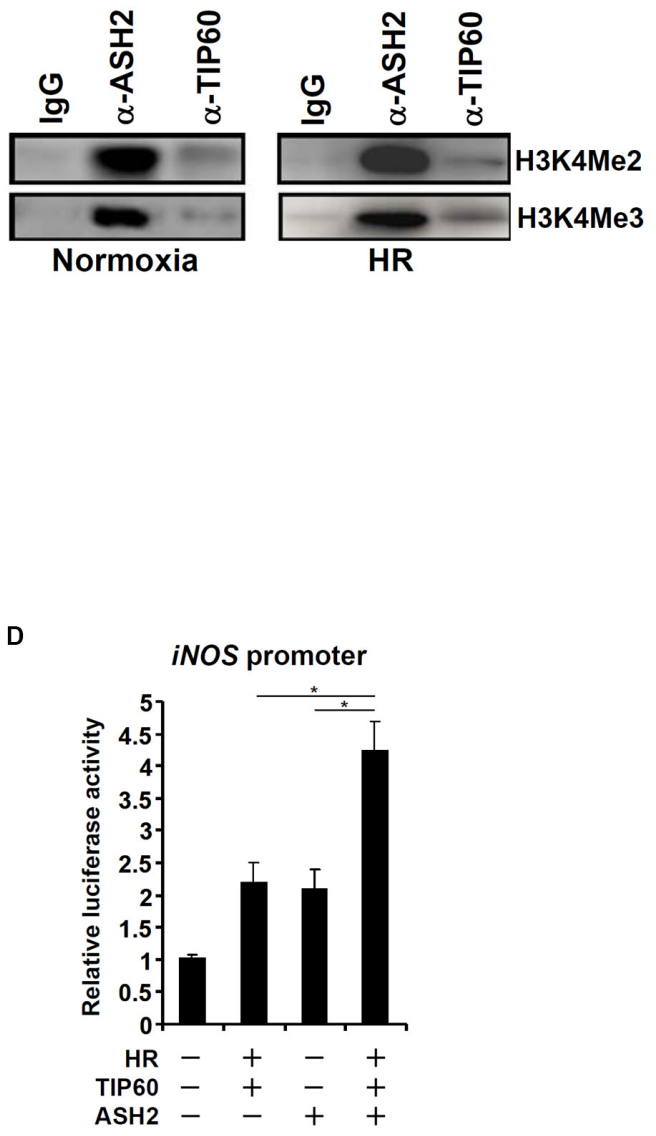

$\mathrm{E}$

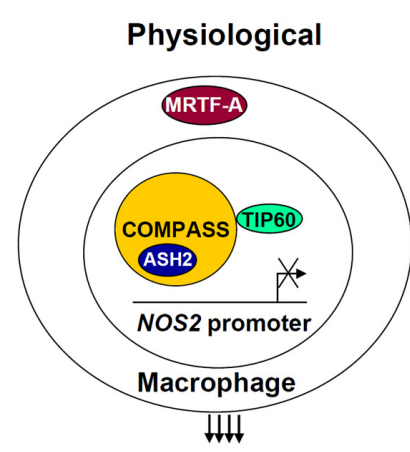

Myocardial homeostasis

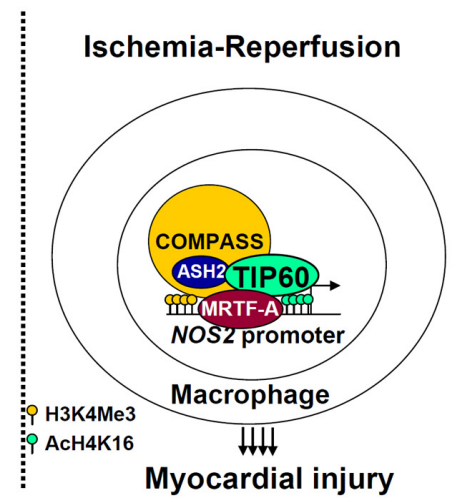

FIGURE 6 | A crosstalk between TIP60 and COMPASS contributes to iNOS transcription. (A) RAW264 cells were transfected with siRNA targeting TIP60 or scrambled siRNA (SCR) followed by exposure to hypoxia-reoxygenation. ChIP assay was performed with anti-trimethyl H3K4. (B) Nuclear lysates from normoxic and HR-stimulated RAW cells were immunoprecipitated with anti-ASH2, anti-TIP60, or lgG. In vitro HMT assay was performed as described in Methods. (C) RAW264 cells were transfected with siRNA targeting TIP60 or scrambled siRNA (SCR) followed by exposure to hypoxia-reoxygenation. Nuclear lysates were

immunoprecipitated with anti-ASH2. In vitro HMT assay was performed as described in Methods. (D) An iNOS promoter construct was transfected into HEK293 cells with TIP60 and/or ASH2. Luciferase activities were normalized by protein concentration and GFP fluorescence. $\mathrm{N}=3$ for all the experiments. Data represent averages of three independent experiments and error bars represent SEM. (E) A schematic model. 


\section{DISCUSSION}

Macrophage-derived pro-inflammatory mediators are critical in the pathogenesis of cardiac IRI. Here we detail a novel epigenetic mechanism whereby a crosstalk between MRTF-A, H4K16 acetyltransferase TIP60, and H3K4 methyltransferase activates iNOS transcription in macrophages (Figure 6E). Mounting evidence suggests that TIP60 plays a key role in the regulation of inflammatory response. For example, it has been shown that an exchange of TIP60 for the co-repressor complex NCoR is responsible for the activation of IL- $1 \beta$ induced NF- $\kappa$ B target genes and the ensuing brain inflammation in neurodegenerative diseases (Baek et al., 2002). TIP60 can also interact with STAT6 and NF- $\kappa \mathrm{B}$ to activate the transcription of $\mathrm{I} \varepsilon$ (encoding $\operatorname{IgE}$ ) in B cells to drive intestinal allergy (Yang G. et al., 2018). On the other hand, several independent investigations suggest that TIP60 acts a co-factor for Foxp3, the master regulator of regulatory $\mathrm{T}$ cells (Tregs), to prevent the pathogenesis of autoimmune diseases by promoting histone H4K16 acetylation on the Foxp3 target promoters and by directly acetylating and stabilizing Foxp3 (Chatila and Williams, 2012). In colorectal cancer cells, TIP60 also exerts an anti-inflammatory response by activating the expression of SUV39H1 and SETDB1, two histone $\mathrm{H} 3 \mathrm{~K} 9$ methyltransferases, which in turn repress the transcription of retrotransposon elements to contain STING/IRF7-mediated inflammation (Rajagopalan et al., 2018). These apparently conflicting reports suggest that TIP60 might contribute to the regulation of cellular inflammation in a cell type- and contextspecific manner. Although our data demonstrate that TIP60 activates iNOS transcription in macrophages, it remains to be determined whether genomewide inflammation-associated transcriptional events are influenced by TIP60. In addition, the benefit of harnessing the TIP60 inhibitor MG149 as a solution to treat reperfusion injury has been weighed against its potential detrimental effects on cardiomyocytes. Fisher et al. (2016) have shown that conditional TIP60 deletion in cardiomyocytes causes cardiac dysfunction and lethality. TIP60 also plays an essential role in the maintenance of stem cell self-renewal and pluripotency (Fazzio et al., 2008). Further studies are clearly warranted to define the precise role of TIP60, beyond being an activator of iNOS transcription, in the pathogenesis of cardiac IRI.

Histone modifying enzymes usually operate within a large protein complex. Our data indicate that TIP60 forms a crosstalk with the H3K4 methyltransferase complex. Consistent with our observation, Stallcup and colleagues have argued that estrogen-induced transcription of estrogen receptor alpha $(\mathrm{ER} \alpha)$ target genes is mediated by ER $\alpha$-dependent recruitment of TIP60, which sequentially recruits the H3K4 methyltransferase MLL1 to catalyze H3K4 monomethylation on the enhancers (Jeong et al., 2011). A provocative observation made by Ayrapetov et al. indicates that SUV39H-mediated H3K9 trimethylation, typically a marker of repressive chromatin, serves to activate TIP60 allowing the DNA repair machinery to fix double-strand break (DSB; Ayrapetov et al., 2014). Similar crosstalk during DSB repair between TIP60 and other histone modifying enzymes including H4K20 methyltransferase (Wang and Goldstein, 2016) and H3K36 methyltransferase
(Li and Wang, 2017) has been proposed. Our data also suggest that TIP60 deficiency compromises the activity of the H3K4 methyltransferase complex although the underlying mechanism remains unclear. It is possible that TIP60 functions as a structural/regulatory component of the $\mathrm{H} 3 \mathrm{~K} 4$ methyltransferase. For instance, depletion of WDR82, a structural component of the H3K4 methyltransferase complex, completely abolishes ASH2-associated $\mathrm{H} 3 \mathrm{~K} 4$ trimethylation without altering $\mathrm{H} 3 \mathrm{~K} 4$ dimethylation (Wu et al., 2008). Additional epigenomic studies should be conducted to address the question as to how the transcription landscape in macrophages is influenced by the communications between TIP60 and the H3K4 methyltransferase complex.

MRTF-A has the reputation of bridging the epigenetic machinery to the basal transcription machinery. ChIP-seq experiments have demonstrated that MRTF-A is responsible for the trimethyl H3K4 landscape in several processes critical to the inflammatory response in macrophages ( $\mathrm{Yu}$ et al., 2017a). We show here that iNOS trans-activation parallels MRTF-A-dependent accumulation of, in addition to acetyl $\mathrm{H} 4 \mathrm{~K} 16$, acetyl $\mathrm{H} 3 \mathrm{~K} 9$, acetyl $\mathrm{H} 3 \mathrm{~K} 27$, and trimethyl $\mathrm{H} 3 \mathrm{~K} 4$ on the iNOS promoter. It remains to be tested whether MRTFA recruits the different modifying enzymes simultaneously or sequentially. It has previously been shown that MRTF-A brokers the interaction between histone methyltransferases (e.g., ASH2) and histone acetyltransferases (e.g., p300) on the endothelin (ET1) promoter (Weng et al., 2015b) and on the collagen type I (COL1A1/COL1A2) promoter (Xu et al., 2015). It would be of interest to examine whether MRTF-A serves as a moderator for the crosstalk between TIP60 and ASH2. Another intriguing yet untested possibility is that MRTF-A might be directly targeted (modified) and thus modulated by TIP60. We have previously shown that a string of four lysine residues within the N-terminus of MRTF-A are subjected to dynamic acetylation in macrophages exposed to pro-inflammatory stimuli (Yu et al., 2017b). Although that study assigned the acetyltransferase PCAF as the major enzyme catalyzing MRTF-A acetylation, TIP60 remains to be determined as a genuine MRTR-A acetyltransferase because it is not uncommon for a single substrate to be targeted by different enzymes.

One of the major limitations of the present study is that it is not clear whether the proposed model applies to macrophages in general or only to lineage-specific macrophages. Here we used an anti-F4/80 antibody to isolate cardiac macrophages from the IR-challenged murine hearts and discovered that MRTF-A deficiency (Figure 1G) or inhibition (Figure 1H) attenuated iNOS expression. It is well known that resident macrophages and circulating monocyte-derived macrophages fulfill distinct functions despite the fact that both populations can be characterized as F4/80 ${ }^{+}$cells (Gosselin et al., 2014; Honold and Nahrendorf, 2018; Link et al., 2018). The development of single cell based sequencing (scRNA-seq) techniques has empowered researchers to more precisely define the molecular signature and functions of specific cell lineages (Hu et al., 2018; Nguyen et al., 2018; Xiao and Guo, 2018; Morrish et al., 2019). Recently, Mold et al. have presented scRNA-seq data to identify unique airspace macrophage subsets in a lung inflammation 
model (Mould et al., 2019). The same technique could be harnessed to verify and validate our model in future studies. Another caveat regarding the present study is its focus on the regulation of macrophage derived iNOS. iNOS can be induced in cardiomyocytes by hypoxia-reoxyenation (Agnetti et al., 2005). Further, cardiomyocyte-specific iNOS expression results in loss of cardiac function and causes sudden death in mice (Mungrue et al., 2002). Whether the same mechanism that contributes to iNOS induction in macrophages as proposed by our model can account for HRinduced iNOS expression in cardiomyocytes remains to be determined. Finally, MG149 has been shown to inhibit TIP60 and another H4K16 acetyltransferase hMOF with comparable potency (Ghizzoni et al., 2012). Therefore, whether the effects of MG149 on iNOS expression were achieved through TIP60 or hMOF or a combination of the two enzymes cannot be ascertained at this point.

In summary, our data unveil a novel epigenetic pathway that may contribute to the pathogenesis of cardiac ischemiareperfusion. Since the small-molecule inhibitor of MRTF$A$ is already available and appears to be effective in animal models (Yu et al., 2018; Li and Xu, 2019), our data provide renewed incentive for targeting MRTF-A in the intervention of cardiac ischemia-reperfusion in the clinics.

\section{DATA AVAILABILITY STATEMENT}

All datasets generated for this study are included in the article/Supplementary Material.

\section{REFERENCES}

Agnetti, G., Bordoni, A., Angeloni, C., Leoncini, E., Guarnieri, C., Caldarera, C. M., et al. (2005). Green tea modulation of inducible nitric oxide synthase in hypoxic/reoxygenated cardiomyocytes. Biochimie 87, 457-460. doi: 10.1016/ j.biochi.2005.01.004

Amberger, A., Schneeberger, S., Hernegger, G., Brandacher, G., Obrist, P., Lackner, P., et al. (2002). Gene expression profiling of prolonged cold ischemia and reperfusion in murine heart transplants. Transplantation 74, 1441-1449. doi: 10.1097/00007890-200211270-00017

Avvakumov, N., and Cote, J. (2007). The MYST family of histone acetyltransferases and their intimate links to cancer. Oncogene 26, 5395-5407. doi: 10.1038/sj.onc. 1210608

Ayrapetov, M. K., Gursoy-Yuzugullu, O., Xu, C., Xu, Y., and Price, B. D. (2014). DNA double-strand breaks promote methylation of histone $\mathrm{H} 3$ on lysine 9 and transient formation of repressive chromatin. Proc. Natl. Acad. Sci. U.S.A. 111, 9169-9174. doi: 10.1073/pnas.1403565111

Baek, S. H., Ohgi, K. A., Rose, D. W., Koo, E. H., Glass, C. K., et al. (2002). Exchange of N-CoR corepressor and Tip60 coactivator complexes links gene expression by NF-kappaB and beta-amyloid precursor protein. Cell 110, 55-67. doi: 10.1016/s0092-8674(02)00809-7

Buxade, M., Lunazzi, G., Minguillon, J., Iborra, S., Berga-Bolanos, R., Del Val, M., et al. (2012). Gene expression induced by Toll-like receptors in macrophages requires the transcription factor NFAT5. J. Exp. Med. 209, 379-393. doi: 10. 1084/jem.20111569

Chatila, T. A., and Williams, C. B. (2012). Foxp3: shades of tolerance. Immunity 36, 693-694. doi: 10.1016/j.immuni.2012.05.011

Chen, D., Yang, Y., Cheng, X., Fang, F., Xu, G., Yuan, Z., et al. (2015). Megakaryocytic leukemia 1 directs a histone H3 lysine 4 methyltransferase

\section{ETHICS STATEMENT}

The animal study was reviewed and approved by Ethics Committee on Humane Treatment of Laboratory Animals of Nanjing Medical University.

\section{AUTHOR CONTRIBUTIONS}

YY, MF, and YX conceived the project. YY, MF, GY, LY, LLin, LLiu, and YX designed experiments. YY and LLin performed all the ChIP assays. YY performed the in vitro HMT assay. GY and LY performed all the animal experiments and collected specimens. MF performed the reporter-luciferase assays. All authors contributed to the QPCR and Western blotting assays. YX wrote the manuscript. MF, YY, GY, and YX secured funding. YY and MF provided supervision.

\section{FUNDING}

This work was supported by grants from the National Natural Science Foundation of China (81870326, 818170302, 81725001, and 81670223; Grant No. 81700221 to GY).

\section{SUPPLEMENTARY MATERIAL}

The Supplementary Material for this article can be found online at: https://www.frontiersin.org/articles/10.3389/fcell.2020.00484/ full\#supplementary-material

complex to regulate hypoxic pulmonary hypertension. Hypertension 65, 821833. doi: 10.1161/hypertensionaha.114.04585

Cheng, X., Yang, Y., Fan, Z., Yu, L., Bai, H., Zhou, B., et al. (2015). MKL1 potentiates lung cancer cell migration and invasion by epigenetically activating MMP9 transcription. Oncogene 34, 5570-5581. doi: 10.1038/onc.2015.14

Crosby, M. B., Svenson, J., Gilkeson, G. S., and Nowling, T. K. (2005). A novel PPAR response element in the murine iNOS promoter. Mol. Immunol. 42, 1303-1310. doi: 10.1016/j.molimm.2004.12.009

Ding, H. L., Zhu, H. F., Dong, J. W., Zhu, W. Z., Yang, W. W., Yang, H. T., et al. (2005). Inducible nitric oxide synthase contributes to intermittent hypoxia against ischemia/reperfusion injury. Acta Pharmacol. Sin. 26, 315-322. doi: 10.1111/j.1745-7254.2005.00046.x

Eltzschig, H. K., and Eckle, T. (2011). Ischemia and reperfusion-from mechanism to translation. Nat. Med. 17, 1391-1401. doi: 10.1038/nm.2507

Fan, Z., Hao, C., Li, M., Dai, X., Qin, H., Li, J., et al. (2015). MKL1 is an epigenetic modulator of TGF-beta induced fibrogenesis. Biochim. Biophys. Acta Gene Regul. Mech. 2015, 1219-1228. doi: 10.1016/j.bbagrm.2015. 07.013

Fan, Z., Li, N., Xu, Z., Wu, J., Fan, X., et al. (2019). An interaction between MKL1. BRG1, and C/EBPbeta mediates palmitate induced CRP transcription in hepatocytes. Biochim. Biophys. Acta Gene Regul. Mech. 1862:194412. doi: 10.1016/j.bbagrm.2019.194412

Fan, Z., Li, Z., Yang, Y., Liu, S., Guo, J., et al. (2017). HIF-1alpha coordinates epigenetic activation of SIAH1 in hepatocytes in response to nutritional stress. Biochim. Biophys. Acta Gene Regul. Mech. 1860, 1037-1046. doi: 10.1016/j. bbagrm.2017.08.002

Fang, F., Yang, Y., Yuan, Z., Gao, Y., Zhou, J., Chen, Q., et al. (2011). Myocardinrelated transcription factor A mediates OxLDL-induced endothelial injury. Circ. Res. 108, 797-807. doi: 10.1161/circresaha.111.240655 
Fazzio, T. G., Huff, J. T., and Panning, B. (2008). An RNAi screen of chromatin proteins identifies Tip60-p400 as a regulator of embryonic stem cell identity. Cell 134, 162-174. doi: 10.1016/j.cell.2008.05.031

Fisher, J. B., Horst, A., Wan, T., Kim, M. S., Auchampach, J., et al. (2016). Depletion of Tip60 from in vivo cardiomyocytes increases myocyte density. followed by cardiac dysfunction, myocyte fallout and lethality. PLoS One 11:e0164855. doi: 10.1371 /journal.pone.0164855

Ghizzoni, M., Wu, J., Gao, T., Haisma, H. J., Dekker, F. J., and George Zheng, Y. (2012). 6-alkylsalicylates are selective Tip60 inhibitors and target the acetylCoA binding site. Eur. J. Med. Chem. 47, 337-344. doi: 10.1016/j.ejmech.2011. 11.001

Gosselin, D., Link, V. M., Romanoski, C. E., Fonseca, G. J., Eichenfield, D. Z., Spann, N. J., et al. (2014). Environment drives selection and function of enhancers controlling tissue-specific macrophage identities. Cell 159, 13271340. doi: 10.1016/j.cell.2014.11.023

Granger, D. N., and Kvietys, P. R. (2015). Reperfusion injury and reactive oxygen species: the evolution of a concept. Redox Biol. 6, 524-551. doi: 10.1016/j.redox. 2015.08.020

Griscavage, J. M., Wilk, S., and Ignarro, L. J. (1996). Inhibitors of the proteasome pathway interfere with induction of nitric oxide synthase in macrophages by blocking activation of transcription factor NF-kappa B. Proc. Natl. Acad. Sci. U.S.A. 93, 3308-3312. doi: 10.1073/pnas.93.8.3308

Honold, L., and Nahrendorf, M. (2018). Resident and monocyte-derived macrophages in cardiovascular disease. Circ. Res. 122, 113-127. doi: 10.1161/ circresaha.117.311071

Hu, Y., An, Q., Sheu, K., Trejo, B., Fan, S., et al. (2018). Single cell multi-omics technology: methodology and application. Front. Cell Dev. Biol 6:28. doi: 10. 3389/fcell.2018.00028

Jeong, K. W., Kim, K., Situ, A. J., Ulmer, T. S., An, W., et al. (2011). Recognition of enhancer element-specific histone methylation by TIP60 in transcriptional activation. Nat. Struct. Mol. Biol. 18, 1358-1365. doi: 10.1038/nsmb.2153

Kong, M., Chen, X., Lv, F., Ren, H., Fan, Z., Qin, H., et al. (2019a). Serum response factor (SRF) promotes ROS generation and hepatic stellate cell activation by epigenetically stimulating NCF1/2 transcription. Redox Biol. 26:101302. doi: 10.1016/j.redox.2019.101302

Kong, M., Hong, W., Shao, Y., Lv, F., Fan, Z., Li, P., et al. (2019b). Ablation of serum response factor in hepatic stellate cells attenuates liver fibrosis. J. Mol. Med. 97, 1521-1533. doi: 10.1007/s00109-019-01831-8

Li, L., and Wang, Y. (2017). Cross-talk between the H3K36me3 and H4K16ac histone epigenetic marks in DNA double-strand break repair. J. Biol. Chem. 292, 11951-11959. doi: 10.1074/jbc.m117.788224

Li, N., Kong, M., Zeng, S., Hao, C., Li, M., Li, L., et al. (2019a). Brahma related gene 1 (Brg1) contributes to liver regeneration by epigenetically activating the Wnt/beta-catenin pathway in mice. FASEB J. 33, 327-338. doi: 10.1096/fj. $201800197 \mathrm{r}$

Li, Z., Chen, B., Dong, W., Kong, M., Fan, Z., Yu, L., et al. (2019b). MKL1 promotes endothelial-to-mesenchymal transition and liver fibrosis by activating TWIST1 transcription. Cell Death Dis. 10:899.

Li, Z., Chen, B., Dong, W., Kong, M., Shao, Y., Fan, Z., et al. (2019c). The chromatin remodeler Brgl integrates ROS production and endothelial-mesenchymal transition to promote liver fibrosis in mice. Front. Dev. Cell Biol. 7:245. doi: 10.3389/fcell.2019.00245

Li, Z., Li, P., Lu, Y., Sun, D., Zhang, X., et al. (2019d). A non-autonomous role of MKL1 in the activation of hepatic stellate cells. Biochim. Biophys. Acta Gene Regul. Mech. 1862, 609-618. doi: 10.1016/j.bbagrm.2019.03.001

Li, Z., Lv, F., Dai, C., Wang, Q., JIang, C., Fang, M., et al. (2019e). Activation of galectin-3 (LGALS3) transcription by injurious stimuli in the liver is commonly mediated by BRG1. Front. Cell Dev. Biol. 7:310. doi: 10.3389/fcell.2019.00310

Li, Z., Xia, J., Fang, M., and Xu, Y. (2019f). Epigenetic regulation of lung cancer cell proliferation and migration by the chromatin remodeling protein BRG1. Oncogenesis 8:66.

Li, N., Kong, M., Zeng, S., Xu, Z., Li, M., Hong, W., et al. (2018a). The chromatin remodeling protein BRG1 regulates APAP-induced liver injury by modulating CYP3A11 transcription in hepatocyte. Biochim. Biophys. Acta Mol. Basis Dis. 1864, 3487-3495. doi: 10.1016/j.bbadis.2018.08.003

Li, N., Li, M., Hong, W., Shao, J., Xu, H., Shimano, H., et al. (2018b). Brg1 regulates pro-lipogenic transcription by modulating SREBP activity in hepatocytes.
Biochim. Biophys. Acta Mol. Basis Dis. 1864, 2881-2889. doi: 10.1016/j.bbadis. 2018.05.022

Li, Z., Chen, B., Dong, W., Xu, W., Song, M., Fang, M., et al. (2018c). Epigenetic activation of PERP transcription by MKL1 contributes to ROS-induced apoptosis in skeletal muscle cells. Biochim. Biophys. Acta Gene Regul. Mech. 1861, 905-915. doi: 10.1016/j.bbagrm.2018.07.011

Li, Z., Chen, B., Weng, X., Yu, L., Song, M., Fang, M., et al. (2018d). The histone methyltransferase SETD1A regulates thrombomodulin transcription in vascular endothelial cells. Biochim. Biophys. Acta Gene Regul. Mech. 1861, 752-761. doi: 10.1016/j.bbagrm.2018.06.004

Li, Z., Zhang, X., Liu, S., Zeng, S., Yu, L., Yang, G., et al. (2018e). BRG1 regulates NOX gene transcription in endothelial cells and contributes to cardiac ischemia-reperfusion injury. Biochim. Biophys. Acta Mol. Basis Dis. 1864, 34773486. doi: 10.1016/j.bbadis.2018.08.002

Li, Z., and Xu, Y. (2019). Response by Li and Xu to Letter Regarding Article, Megakaryocytic Leukemia 1 Bridges Epigenetic Acivation of NADPH Oxidase in macrophages to cardiac ischemia-reperfusion injury. Circulation 139, e965e966.

Lin, S. Y., Li, T. Y., Liu, Q., Zhang, C., Li, X., Chen, Y., et al. (2012). GSK3-TIP60ULK1 signaling pathway links growth factor deprivation to autophagy. Science 336, 477-481. doi: 10.1126/science.1217032

Ling, H., Edelstein, C., Gengaro, P., Meng, X., Lucia, S., Knotek, M., et al. (1999). Attenuation of renal ischemia-reperfusion injury in inducible nitric oxide synthase knockout mice. Am. J. Physiol. 277, F383-F390.

Link, V. M., Duttke, S. H., Chun, H. B., Holtman, I. R., Westin, E., Hoeksema, M. A., et al. (2018). Analysis of Genetically Diverse Macrophages Reveals Local and Domain-wide Mechanisms that Control Transcription Factor Binding and Function. Cell 173:e17.

Liu, L., Hong, W., Li, M., Ren, H., Wang, J., Xu, H., et al. (2019a). A Cross Talk Between BRG1 and Males absent on the first contributes to reactive oxygen species production in a mouse model of nonalcoholic Steatohepatitis. Antioxid. Redox. Signal. 30, 1539-1552. doi: 10.1089/ars.2016.6822

Liu, L., Mao, L., Wu, X., Wu, T., Liu, W., Yang, Y., et al. (2019b). BRG1 regulates endothelial-derived IL-33 to promote ischemia-reperfusion induced renal injury and fibrosis in mice. Biochim. Biophys. Acta Mol. Basis Dis. 1865, 2551-2561. doi: 10.1016/j.bbadis.2019.06.015

Liu, L., Wu, X., Xu, H., Yu, L., Zhang, X., Li, L., et al. (2018). Myocardinrelated transcription factor A (MRTF-A) contributes to acute kidney injury by regulating macrophage ROS production. Biochim. Biophys. Acta Mol. Basis Dis. 1864, 3109-3121. doi: 10.1016/j.bbadis.2018.05.026

Lowenstein, C. J., Alley, E. W., Raval, P., Snowman, A. M., Snyder, S. H., Russell, S. W., et al. (1993). Macrophage nitric oxide synthase gene: two upstream regions mediate induction by interferon gamma and lipopolysaccharide. Proc. Natl. Acad. Sci. U.S.A. 90, 9730-9734. doi: 10.1073/pnas.90.20.9730

Lu, Y., Lv, F., Kong, M., Chen, X., Duan, Y., Sun, D., et al. (2019). A cAbl-MRTF-a feedback loop contributes to hepatic stellate cell activation. Front. Cell Dev. Biol. 7:243. doi: 10.3389/fcell.2019.00243

Luchsinger, L. L., Patenaude, C. A., Smith, B. D., and Layne, M. D. (2011). Myocardin-related transcription factor-A complexes activate type I collagen expression in lung fibroblasts. J. Biol. Chem. 286, 44116-44125. doi: 10.1074/ jbc.m111.276931

Lutz, J., Thurmel, K., and Heemann, U. (2010). Anti-inflammatory treatment strategies for ischemia/reperfusion injury in transplantation. J. Inflamm. 7:27. doi: 10.1186/1476-9255-7-27

Marfella, R., Di Filippo, C., Esposito, K., Nappo, F., Piegari, E., Cuzzocrea, S., et al. (2004). Absence of inducible nitric oxide synthase reduces myocardial damage during ischemia reperfusion in streptozotocin-induced hyperglycemic mice. Diabetes Metab. Res. Rev. 53, 454-462. doi: 10.2337/diabetes.53.2.454

Morita, T., Mayanagi, T., and Sobue, K. (2007). Dual roles of myocardin-related transcription factors in epithelial mesenchymal transition via slug induction and actin remodeling. J. Cell Biol. 179, 1027-1042. doi: 10.1083/jcb.200708174

Morrish, R. B., Hermes, M., Metz, J., Stone, N., Pagliara, S., Chahwan, R., et al. (2019). Single cell imaging of nuclear architecture changes. Front. Cell Dev. Biol. 7:141. doi: 10.3389/fcell.2019.00141

Mould, K. J., Jackson, N. D., Henson, P. M., Seibold, M., and Janssen, W. J. (2019). Single cell RNA sequencing identifies unique inflammatory airspace macrophage subsets. JCI Insight 4:e126556. doi: 10.1172/jci.insight.126556 
Mungrue, I. N., Gros, R., You, X., Pirani, A., Azad, A., Csont, T., et al. (2002). Cardiomyocyte overexpression of iNOS in mice results in peroxynitrite generation, heart block, and sudden death. J. Clin. Invest. 109, 735-743. doi: 10.1172/jci0213265

Nguyen, Q. H., Pervolarakis, N., Nee, K., and Kessenbrock, K. (2018). Experimental considerations for single-cell RNA sequencing approaches. Front. Cell Dev. Biol. 6:108. doi: 10.3389/fcell.2018.00108

Rajagopalan, D., Tirado-Magallanes, R., Bhatia, S. S., Teo, W. S., Sian, S., Hora, S., et al. (2018). TIP60 represses activation of endogenous retroviral elements. Nucleic Acids Res. 46, 9456-9470. doi: 10.1093/nar/gky659

Ren, D., Wang, X., Ha, T., Liu, L., Kalbfleisch, J., Gao, X., et al. (2013). SRA deficiency reduces myocardial ischemia/reperfusion injury; involvement of increased microRNA-125b expression in macrophages. Biochim. Biophys. Acta 1832, 336-346. doi: 10.1016/j.bbadis.2012.10.012

Shao, J., Weng, X., Zhuo, L., Yu, L., Li, Z., Shen, K., et al. (2019). Angiotensin II induced CSF1 transcription is mediated by a crosstalk between different epigenetic factors in vascular endothelial cells. Biochim. Biophys. Acta Gene Regul. Mech. 1862, 1-11. doi: 10.1016/j.bbagrm.2018.10.001

Shilatifard, A. (2012). The COMPASS family of histone H3K4 methylases: mechanisms of regulation in development and disease pathogenesis. Annu. Rev. Biochem. 81, 65-95. doi: 10.1146/annurev-biochem-051710-134100

Small, E. M., Thatcher, J. E., Sutherland, L. B., Kinoshita, H., Gerard, R. D., Richardson, J. A., et al. (2010). Myocardin-related transcription factor-a controls myofibroblast activation and fibrosis in response to myocardial infarction. Circ. Res. 107, 294-304. doi: 10.1161/circresaha.110.223172

Sun, Y., Boyd, K., Xu, W., Ma, J., Jackson, C. W., Fu, A., et al. (2006). Acute myeloid leukemia-associated Mkll (Mrtf-a) is a key regulator of mammary gland function. Mol. Cell. Biol. 26, 5809-5826. doi: 10.1128/mcb.00024-06

Suzuki, Y., Deitch, E. A., Mishima, S., Lu, Q., and Xu, D. (2000). Inducible nitric oxide synthase gene knockout mice have increased resistance to gut injury and bacterial translocation after an intestinal ischemia-reperfusion injury. Crit. Care Med. 28, 3692-3696. doi: 10.1097/00003246-200011000-00026

Taipale, M., Rea, S., Richter, K., Vilar, A., Lichter, P., Imhof, A., et al. (2005). hMOF histone acetyltransferase is required for histone $\mathrm{H} 4$ lysine 16 acetylation in mammalian cells. Mol. Cell. Biol. 25, 6798-6810. doi: 10.1128/mcb.25.15.67986810.2005

Tang, J., Cho, N. W., Cui, G., Manion, E. M., Shanbhag, N. M., Botuyan, M. V., et al. (2013). Acetylation limits 53BP1 association with damaged chromatin to promote homologous recombination. Nat. Struct. Mol. Biol. 20, 317-325. doi: $10.1038 / \mathrm{nsmb} .2499$

Tian, W., Hao, C., Fan, Z., Weng, X., Qin, H., Wu, X., et al. (2015). Myocardin related transcription factor A programs epigenetic activation of hepatic stellate cells. J. Hepatol. 62, 165-174. doi: 10.1016/j.jhep.2014.07.029

Tompkins, A. J., Burwell, L. S., Digerness, S. B., Zaragoza, C., Holman, W. L., et al. (2006). Mitochondrial dysfunction in cardiac ischemia-reperfusion injury: ROS from complex I, without inhibition. Biochim. Biophys. Acta 1762, 223-231. doi: 10.1016/j.bbadis.2005.10.001

Wang, Q., and Goldstein, M. (2016). Small RNAs Recruit Chromatin-Modifying Enzymes MMSET and Tip60 to reconfigure damaged DNA upon double-strand break and facilitate repair. Cancer Res. 76, 1904-1915. doi: 10.1158/0008-5472. can-15-2334

Weng, X., Yu, L., Liang, P., Chen, D., Cheng, X., Yang, Y., et al. (2015a). Endothelial MRTF-A mediates angiotensin II induced cardiac hypertrophy. J. Mol. Cell Cardiol. 80, 23-33.

Weng, X., Yu, L., Liang, P., Li, L., Dai, X., Zhou, B., et al. (2015b). A crosstalk between chromatin remodeling and histone $\mathrm{H} 3 \mathrm{~K} 4$ methyltransferase complexes in endothelial cells regulates angiotensin II-induced cardiac hypertrophy. J. Mol. Cell Cardiol. 82, 48-58.

Weng, X., Zhang, Y., Li, Z., Yu, L., Xu, F., Fang, M., et al. (2019). Class II transactivator (CIITA) mediates IFN-gamma induced eNOS repression by enlisting SUV39H1. Biochim. Biophys. Acta Gene Regul. Mech. 1862, 163-172.
Wu, M., Wang, P. F., Lee, J. S., Martin-Brown, S., Florens, L., Washburn, M., et al. (2008). Molecular regulation of H3K4 trimethylation by Wdr82, a component of human Set1/COMPASS. Mol. Cell. Biol. 28, 7337-7344.

Xiao, L., and Guo, J. (2018). Single-Cell in Situ RNA analysis with switchable fluorescent oligonucleotides. Front. Cell Dev. Biol. 6:42. doi: 10.3389/fcell.2018. 00042

Xie, Q. W., Whisnant, R., and Nathan, C. (1993). Promoter of the mouse gene encoding calcium-independent nitric oxide synthase confers inducibility by interferon gamma and bacterial lipopolysaccharide. J. Exp. Med. 177, 17791784.

Xu, H., Wu, X., Qin, H., Tian, W., Chen, J., Sun, L., et al. (2015). Myocardinrelated transcription factor a epigenetically regulates renal fibrosis in diabetic nephropathy. J. Am. Soc. Nephrol. 26, 1648-1660.

Yang, G., Cheng, B. H., Yang, S. B., Liu, Z. Q., Qiu, S. Q., Yang, L. T., et al. (2018). Targeting histone-acetyltransferase Tat-interactive protein 60 inhibits intestinal allergy. Allergy 73, 387-394.

Yang, Y., Li, X., Peng, L., An, L., Sun, N., Hu, X., et al. (2018). Tanshindiol C inhibits oxidized low-density lipoprotein induced macrophage foam cell formation via a peroxiredoxin 1 dependent pathway. Biochim. Biophys. Acta Mol. Basis Dis. 1864, 882-890.

Yang, Y., Liu, L., Fang, M., Bai, H., and Xu, Y. (2019a). The chromatin remodeling protein BRM regulates the transcription of tight junction proteins: implication in breast cancer metastasis. Biochim. Biophys. Acta Gene Regul. Mech. 1862, 547-556.

Yang, Y., Liu, L., Li, M., Cheng, X., Fang, M., Zeng, Q., et al. (2019b). The chromatin remodeling protein BRG1 links ELOVL3 trans-activation to prostate cancer metastasis. Biochim. Biophys. Acta Gene Regul. Mech. 1862, 834-845.

Yu, L., Fang, F., Dai, X., Xu, H., Qi, X., Fang, M., et al. (2017a). MKL1 defines the H3K4Me3 landscape for NF-kappaB dependent inflammatory response. Sci. Rep. 7:191.

Yu, L., Li, Z., Fang, M., and Xu, Y. (2017b). Acetylation of MKL1 by PCAF regulates pro-inflammatory transcription. Biochim. Biophys. Acta Gene Regul. Mech. 1860, 839-847.

Yu, L., Weng, X., Liang, P., Dai, X., Wu, X., Xu, H., et al. (2014). MRTF-A mediates LPS-induced pro-inflammatory transcription by interacting with the COMPASS complex. J. Cell Sci. 127, 4645-4657.

Yu, L., Yang, G., Zhang, X., Wang, P., Weng, X., Yang, Y., et al. (2018). Megakaryocytic Leukemia 1 (MKL1) Bridges Epigenetic Activation of NADPH Oxidase in macrophages to cardiac ischemia-reperfusion injury. Circulation $138,2820-2836$.

Zeng, S., Wu, X., Chen, X., Xu, H., Zhang, T., et al. (2018). Hypermethylated in cancer 1 (HIC1) mediates high glucose induced ROS accumulation in renal tubular epithelial cells by epigenetically repressing SIRT1 transcription. Biochim. Biophys. Acta Gene Regul. Mech. 1861, 917-927.

Zhang, X., Liu, S., Weng, X., Wu, T., Yu, L., Xu, Y., et al. (2018). Brg1 trans-activates endothelium-derived colony stimulating factor to promote calcium chloride induced abdominal aortic aneurysm in mice. J. Mol. Cell Cardiol. 125, 6-17.

Zhao, Q., Yang, J., Chen, H., Li, J., Que, L., Zhu, G., et al. (2019). Peli1 induction impairs cardiac microvascular endothelium through Hsp90 dissociation from IRE1alpha. Biochim. Biophys. Acta Mol. Basis Dis. 1865, 2606-2617.

Conflict of Interest: The authors declare that the research was conducted in the absence of any commercial or financial relationships that could be construed as a potential conflict of interest.

Copyright (C) 2020 Yang, Yang, Yu, Lin, Liu, Fang and Xu. This is an open-access article distributed under the terms of the Creative Commons Attribution License (CC BY). The use, distribution or reproduction in other forums is permitted, provided the original author(s) and the copyright owner(s) are credited and that the original publication in this journal is cited, in accordance with accepted academic practice. No use, distribution or reproduction is permitted which does not comply with these terms. 